\title{
DETERMINACIÓN DE LAS ETAPAS DE INICIO DE MACOLLAMIENTO, INICIO DE PRIMORDIO, FLORACIÓN Y MADUREZ EN LA PLANTA DE ARROZ, CON EL SISTEMA S, V Y R CORRELACIONADO CON LA SUMATORIA TÉRMICA
}

\author{
Jennifer Velázquez, ${ }^{*}$ Andrea Rosales ${ }^{*}$, Hernán Rodríguez $^{*}$, Rafael Salas $^{1 / * *}$ \\ Palabras clave: Arroz; fenología; temperatura; grados día; macollamiento; primordio; número de hojas. \\ Keywords: Rice; phenology; temperature; degree day; tillering; primordium; leaf number.
}

\begin{abstract}
RESUMEN
En el cultivo del arroz, la temperatura es uno de los factores de clima que ejerce mayor efecto sobre el crecimiento, desarrollo y producción de la planta; la acumulación de calor constituye la energía necesaria para completar un determinado estado fenológico. Se determinó en plantas de arroz las etapas de inicio de macollamiento, inicio de primordio, floración y madurez de cosecha, empleando el sistema S, V y $\mathrm{R}$, propuesto recientemente por Counce et ál. (2000), que consiste en el conteo del número de hojas completamente desarrolladas; además, se correlacionó con los grados día acumulados que la planta presentaba en ese momento, para estimar con cuántos grados día se iniciaba una etapa fenológica; este parámetro está relacionado con la temperatura promedio diaria y una temperatura base de $10^{\circ} \mathrm{C}$. Para el inicio de macollamiento la planta necesitó de 140,9 grados día; en inicio de primordio 1268,9; para floración 1746,0; y completó su ciclo con un total de 2333,2 grados día. El estudio permite concluir que, para una variedad de ciclo largo (130-135 días), cuando la acumulación de grados día sea igual o similar a los datos anteriores, la planta está iniciando una de las etapas fenológicas mencionadas; no
\end{abstract}

1 Autor para correspondencia. Correo electrónico: rafael.salas@ucr.ac.cr

* Hacienda Mojica, Bagaces, Guanacaste, Costa Rica.

\begin{abstract}
Determining the stages of tillering stage, initiation of primordia, flowering and maturity in the rice plant, with the system $S, V$ and $R$ correlated with the thermal sum at the time. Temperature is one of the major climatic factors that affect growth, development and yield of the rice crop, and also can reduce the time of change of phenological stages. The beginning stages of tillering, initiation of primordia, flowering and harvest maturity were determined with the $\mathrm{S}, \mathrm{V}$ and $\mathrm{R}$ system recently proposed by Counce et ál. (2000); it consists on counting the number of fully developed leaves; in addition, a correlation was made with accumulated degree days that the plant had at that time, in order to estimate with how many degree days the plant began a phenological stage; this parameter is related to the average daily temperature and a base temperature of $10^{\circ} \mathrm{C}$. For the start of tillering the plant needed 140.9 degree days; for primordium start, 1268.9; for bloom 1746; and completed its cycle with a total of 2333.2 degree days. This allows to conclude that, for a variety of long cycle (130-135 days), when the accumulation of degree days is equal or similar to the previous data, the plant initiates one of the above-mentioned phenological
\end{abstract}

\footnotetext{
** Centro de Investigaciones Agronómicas. Universidad de Costa Rica, San José, Costa Rica.
} 
obstante, se debe calibrar cada una de las variedades de mayor uso por el productor, debido a que entre ellas existen diferencias en duración del ciclo de cultivo.

\section{INTRODUCCIÓN}

En los últimos años se ha expuesto que la planta de arroz alcanza una etapa vegetativa a cierto número de días después de germinado, no obstante, en la actualidad se ha estudiado que existen factores climáticos como la temperatura máxima y mínima, las cuales influyen en el desarrollo de la planta de arroz. Toda aceleración o disminución del ritmo vegetativo o de la velocidad de desarrollo, regido por la temperatura, afecta al cultivo aspecto que provoca variaciones en su rendimiento (Saumell 1980, Nishiyama 1976, Yoshida 1981, Streck et ál. 2003).

Villalobos y Retana (1997) exponen que la temperatura afecta la floración y otros estados de desarrollo de las plantas y que la reducción del rendimiento se debe al tiempo térmico diario o grados días, los cuales se acumulan más rápidamente conforme aumenta la temperatura, lo que ocasiona que se acorte el período entre la siembra y la diferenciación floral.

La temperatura promedio diaria determina el ritmo de crecimiento y modifica también el ciclo de cada variedad, debido a que cada etapa fenológica necesita la acumulación de una determinada cantidad de unidades térmicas (Pascale y Damario 2004, Méndez 2002). El arroz necesita para germinar un mínimo de 10 a $13^{\circ} \mathrm{C}$, considerándose su óptimo entre 30 y $35^{\circ} \mathrm{C}$. Por encima de $40^{\circ} \mathrm{C}$ no se produce la germinación. El crecimiento del tallo, hojas y raíces tiene un mínimo de $7^{\circ} \mathrm{C}$, considerándose su óptimo en $\operatorname{los} 23^{\circ} \mathrm{C}$. Con temperaturas superiores a ésta, las plantas crecen más rápidamente, pero los tejidos se hacen más stages; however, each one of the varieties in use by farmers must be calibrated, because there are differences in crop cycle length among them.

blandos, lo que los hace más susceptibles a los ataques de plagas y enfermedades (Datta 1986).

La acción positiva que la temperatura ejerce sobre el crecimiento y desarrollo vegetal se expresa, mediante distintos índices, como sumatoria de unidades térmicas o grados-día. Existen diversas formas para calcular los grados día. En general, la más simple es la del método del promedio. En este caso, se suma la temperatura máxima y mínima del día y el resultado se divide por 2. A este nuevo valor se le resta la temperatura umbral o límite inferior, la cual por ejemplo en arroz es de $10^{\circ} \mathrm{C}$ (Maldonado et ál. 2006, Steinmetz et ál. 2007) y constituye un mejor descriptor que el tiempo biológico con la utilización de días calendario.

En el cultivo del arroz se han desarrollado diversos sistemas que describen el desarrollo fenológico de la planta: Zadoks et ál. (1974), IRRI (1980), Lancashire et ál. (1991), Haun (1973), Counce et ál. (2000). Dentro de estos sistemas, el número de hojas en el tallo principal ha sido el más utilizado (Langer 1972). La importancia de estimar la duración de distintas fases fenológicas radica en que muchas de las actividades en la producción de arroz que permiten aumentar los rendimientos están correlacionados con las etapas de desarrollo; como es el caso de las fertilizaciones, que se realizan básicamente cuando el arroz está en inicio de macollamiento y en diferenciación de primordio.

El conocimiento del desarrollo fenológico de la planta de arroz junto con la acumulación de grados día es una metodología que permite un mejor manejo cultural del cultivo. Esta metodología requiere determinar de manera precisa el 
momento en que la planta inicia o alcanza una etapa. Para ello Counce et ál. (2000), propusieron dividir el desarrollo de la planta en 3 etapas fenológicas: etapa de plántula que va desde la siembra hasta la emergencia de la planta; etapa vegetativa que va de la emergencia hasta la aparición de la hoja bandera y la etapa reproductiva que va desde la diferenciación de panícula hasta la madurez fisiológica; cada una de estas etapas se identifican por la terminología $\mathrm{S}, \mathrm{V}$ y $\mathrm{R}$, respectivamente. Cada etapa fenológica a su vez, se relaciona con el número de hojas que la planta presente en ese determinado momento.

El sistema de conteo de números de hojas determinados para la etapa S (S0 a S3), es una metodología que describe los sucesos que ocurren en la germinación ya sea por la aparición del coleoptilo o la radícula. La primera hoja rudimentaria emerge del coleoptilo y se denomina profilo y no tiene lámina, por eso no se considera una hoja verdadera (Yoshida 1981). Seguidamente aparece la primera hoja completa con vaina, cuello y lámina, una vez emergida se inicia con la terminología V (V1 a V13), el número que presenta la $\mathrm{V}$ va a indicar el total de hojas completamente desarrolladas así mismo como el inicio y final de una etapa. Por ejemplo cuando la planta de arroz presenta una hoja completamente desarrollada se puede mencionar que está en V1. Cuando se tiene 4 hojas completamente desarrolladas o V4 la planta ha iniciado su macollamiento y cuando está en V9 ha iniciado la diferenciación de primordio, la cual a su vez inicia la fase reproductiva y se implementa la terminología R ( $\mathrm{R}$ a R9). En la etapa denominada $\mathrm{R} 4$ la planta se encuentra en floración, en R6 el grano está lechoso, en R7 el grano pastoso y en R9 el grano está para cosecha. Al combinar el conteo de números de hojas y $\mathrm{R}$ y relacionarlos con los grados día acumulados, se puede observar el efecto del factor temperatura en el desarrollo fenológico de la planta de arroz.

Este trabajo tuvo como objetivo determinar el estado fenológico de la planta de arroz por medio del sistema S, V y R y correlacionar los momentos en que inicia una etapa de desarrollo con los grados día acumulados en ese instante para definir el momento oportuno de realizar alguna práctica de manejo del cultivo.

\section{MATERIALES Y MÉTODOS}

La investigación se llevó a cabo en Hacienda Mojica, ubicada en el cantón Bagaces, Guanacaste. Latitud $10^{\circ} 24^{\prime}$, longitud $85^{\circ} 10^{\prime}$.

La variedad que se utilizó fue Fedearroz 50, la cual tiene un ciclo de 130 días.

El área total de la práctica fue de $3000 \mathrm{~m}^{2}$.

Se realizó el conteo de las hojas o V completamente desarrolladas en 16 puntos de muestreo, las valoraciones se hicieron semanalmente durante todo el ciclo de la variedad. Con esta variable se determinó el número de la $\mathrm{V}$ total con la que la planta completa una etapa por medio del sistema propuesto por Counce et ál. (2000) el cual se presenta en la Figura 1. 


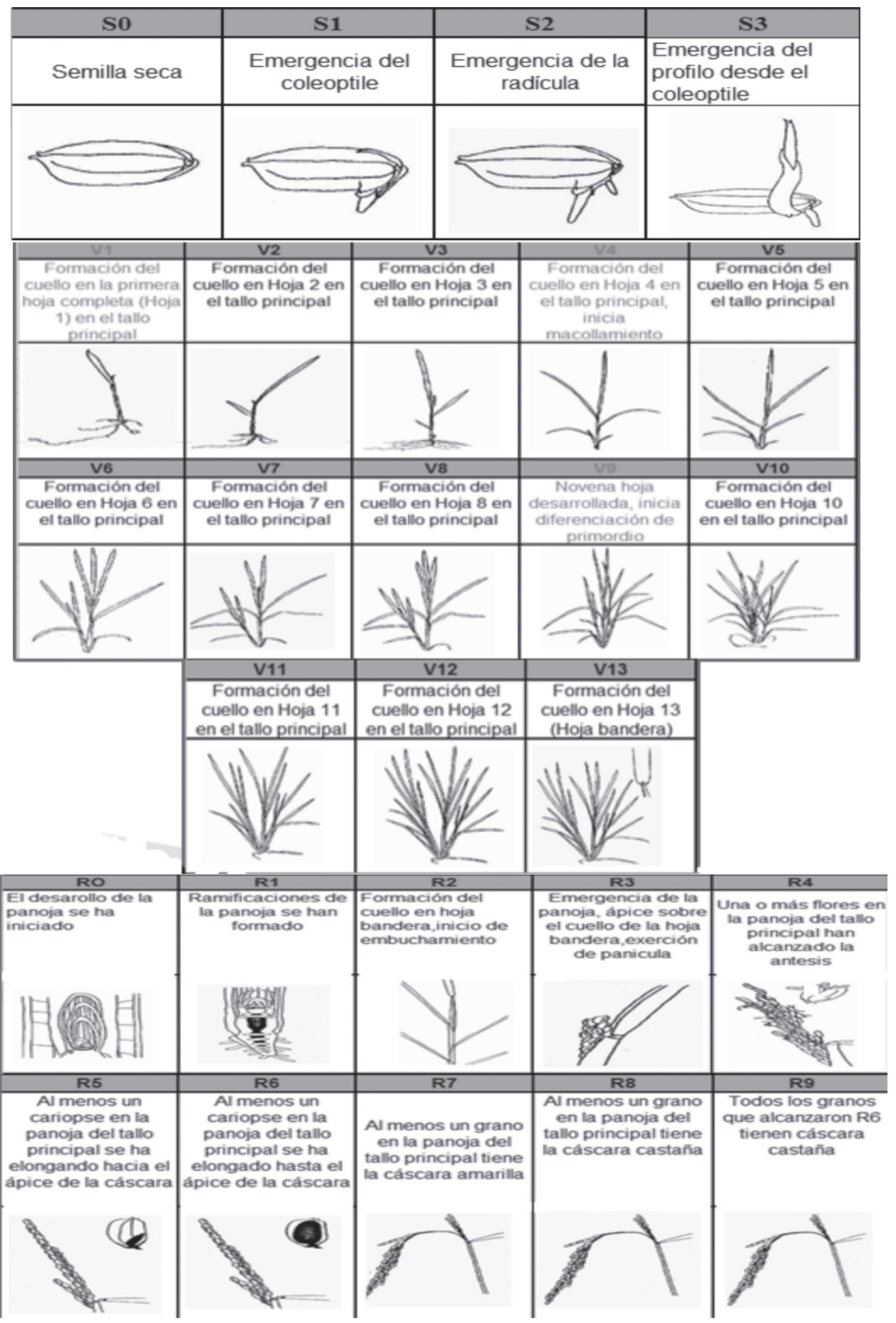

Fig. 1. Descripción de los estados fenológicos de la planta de arroz. Tomado de Counce et ál. 2000. 
Las principales etapas que se evaluaron fueron: inicio de macollamiento, diferenciación de primordio, floración y madurez de cosecha.

La sumatoria térmica o grados día fue calculada por el método de Arnold (1960). Los datos de temperatura para calcular los grados días fueron extraídos de la estación meteorológica del Ingenio Taboga S. A., y la fórmula que se utilizó fue la siguiente:

Grados días Acumulados $=\sum^{\mathrm{n}}(($ Temp. Máx + Temp. Mín) / 2) - Temp. Base *
*Donde la temperatura base para el cultivo de arroz es de $10^{\circ} \mathrm{C}$ y n el número de días en el período considerado (Etapas fenológicas).

\section{RESULTADOS Y DISCUSIÓN}

El inicio de las principales etapas de desarrollo del cultivo de arroz según el conteo de número de hojas se muestra en el Cuadro 1, la etapa de macollamiento (V4, Figura 2) inició a

Cuadro 1. Determinación de las etapas de macollamiento, primordio, floración y madurez de la planta de arroz según el número de hojas.

\begin{tabular}{lcc}
\hline ETAPA (V) & FECHA & DDG $^{*}$ \\
\hline Siembra & $27 / 12 / 2009$ & 0 \\
Germinación & $01 / 01 / 2010$ & 0 \\
Macollamiento (V4) & $08 / 01 / 2010$ & 8 \\
Pimordio (V9) & $11 / 03 / 2010$ & 70 \\
Floración (R4) & $04 / 04 / 2010$ & 94 \\
Cosecha (R9) & $05 / 05 / 2010$ & 125 \\
\hline
\end{tabular}

* DDG : Días después de germinado.

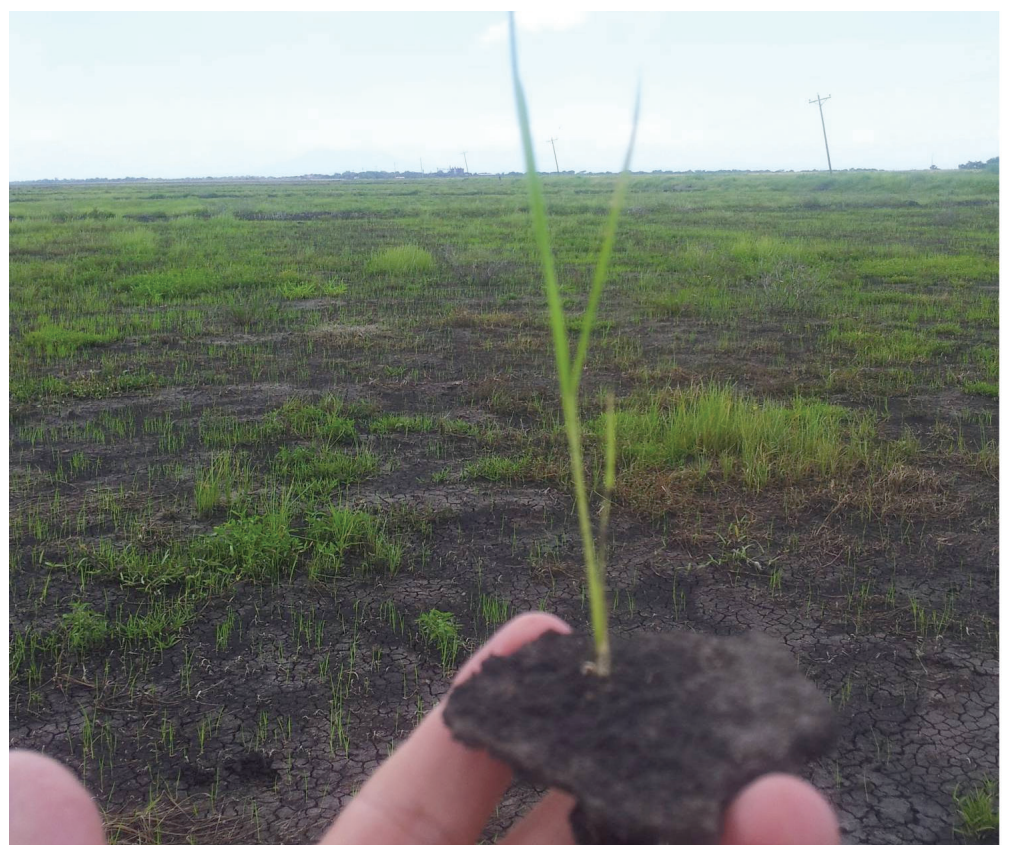

Fig. 2. Etapa fenológica V3 - V4 (Inicio de macollamiento). 
los 8 días después de haber germinado, la diferenciación de primordio (V9, Figura 3) se dio a los 70 días, la floración ( R4, Figura 4) a los 94 días y la madurez de cosecha (R9, Figura 5) a los 125 días, si se comparan los resultados con los días en los que se dice que la planta de arroz inicia

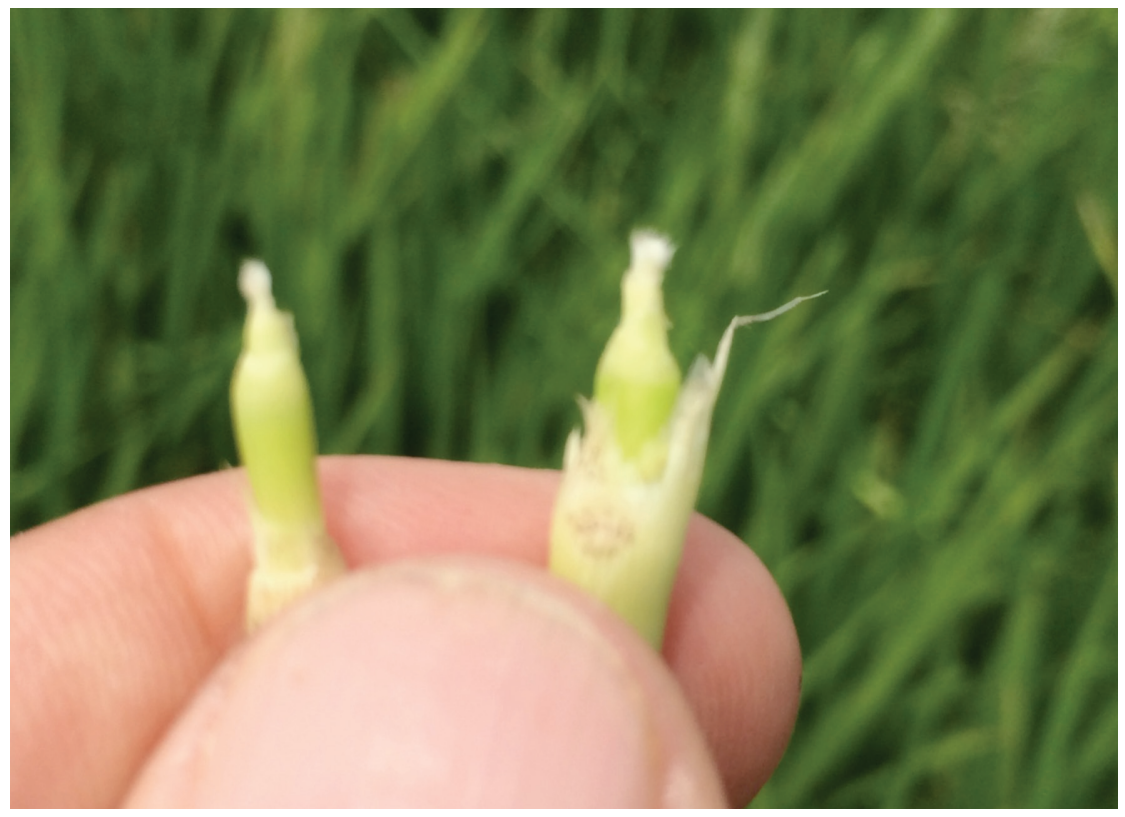

Fig. 3. Diferenciación de panícula. Etapa V9 - 10 (Fase reproductiva).

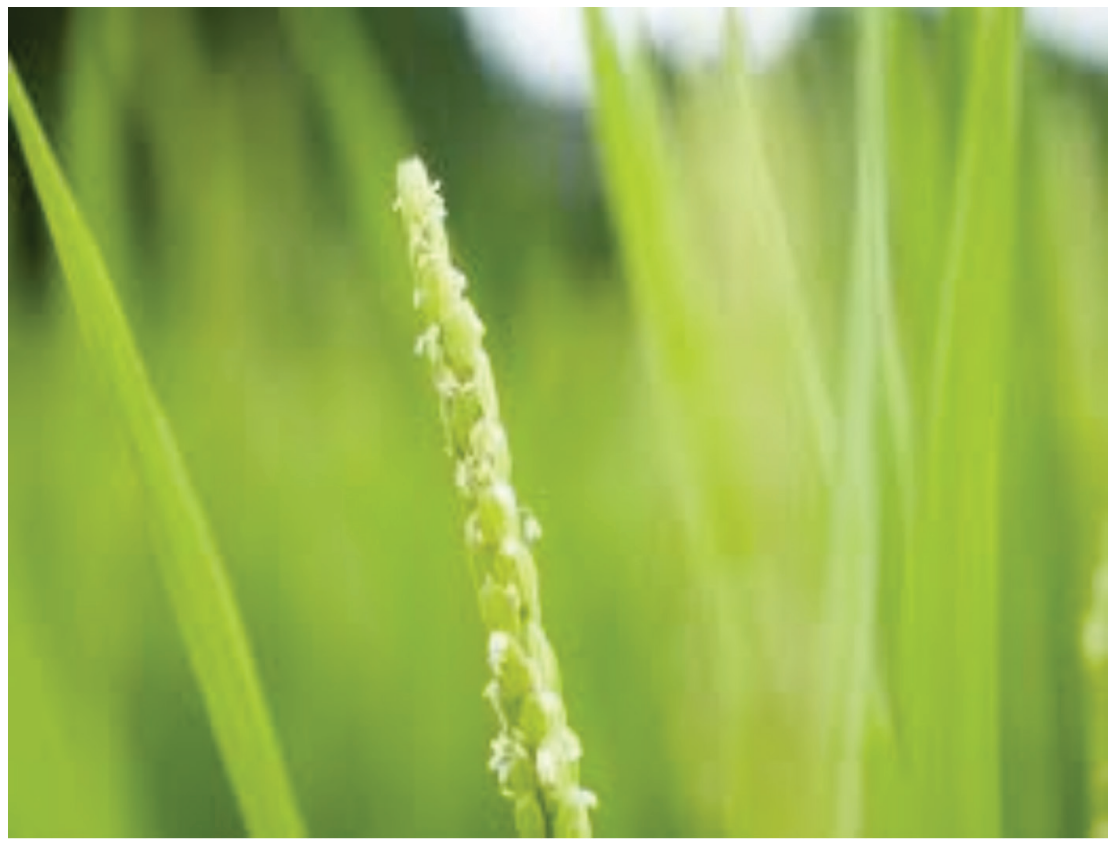

Fig. 4. Floración. Etapa R4. 


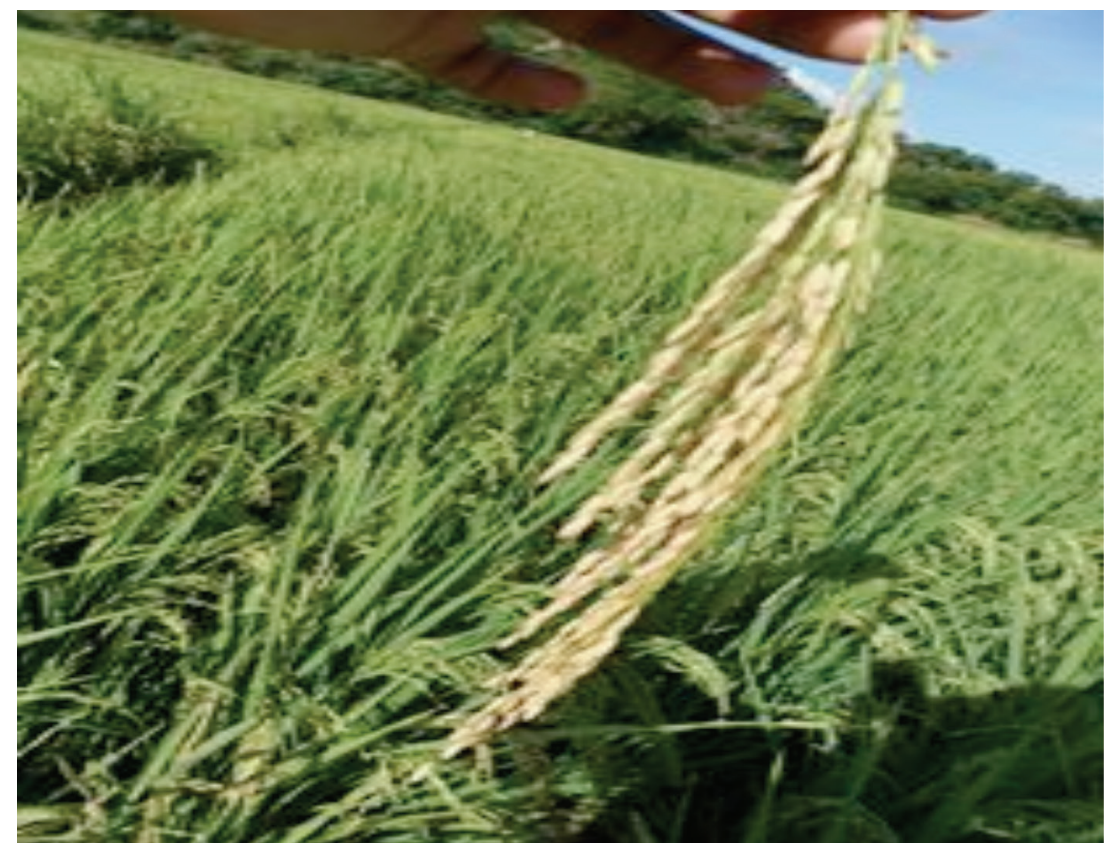

Fig. 5. Maduración. Etapa R9.

una nueva etapa fenológica, se puede observar que existe una reducción de número de días para la etapa vegetativa comprendida de emergencia a macollamiento. La temperatura presente durante el período de germinación hasta el final del macollamiento pudo haber influido en la reducción en días de dicha fase vegetativa, la planta posiblemente logró acumular una cierta cantidad de grados días que le permitió adelantar este proceso. Esta reducción de la etapa vegetativa ya había sido reportada por Steinmetz (2007) quien indica que en escenarios de altas temperaturas en la fase vegetativa de germinación a final de macollamiento, se reduce el desarrollo de la planta (menor número de tallos por cepa y de área foliar) y su rendimiento debido a un incremento en la respiración de la planta. Con respecto al inicio de las demás fases de desarrollo se logra observar que no hubo mayor alteración, porque la planta de arroz terminó su ciclo en los días establecidos para la variedad.

Después de haber determinado el momento en que la planta de arroz inicia una etapa de desarrollo con el método de Counce et ál. (2000), se relacionó dichas etapas fenológicas con la sumatoria de grados días; el Cuadro 2 presenta la suma térmica o grados días acumulados en cada etapa evaluada.

Cuadro 2. Determinación de los grados días en las etapas de macollamiento, primordio, floración y madurez de la planta de arroz.

\begin{tabular}{clc}
\hline Etapa de Desarrollo & \multicolumn{1}{c}{ Descripción } & Grados Días Acumulados \\
\hline V4 & Inicio de Macollamiento & 140,9 \\
V9 & Inicio de Primordio & 1268,9 \\
R4 & Floración & 1746,0 \\
R9 & Madurez de Cosecha & 2333,2 \\
\hline
\end{tabular}


Para la etapa de inicio de macollamiento o V4, la planta tenía un acumulado de 140,9 grados días, en inicio de primordio aumentó a 1268,9, en floración necesitó de 1746; y completó su ciclo con un total de 2333,2 grados días.

Una importante aplicación de esta información será la determinación de cuantos grados días necesita una planta para alcanzar una etapa de desarrollo y permitirá llevar un monitoreo más certero para realizar las prácticas de manejo del cultivo en el momento que más lo requiera, porque se relacionaría su desarrollo directamente con una variable de clima que hasta el momento no había sido utilizada.

Como lo menciona Méndez (2002), las variedades de arroz poseen diferentes requerimientos de suma térmica para alcanzar los diferentes estados fenológicos. Con la variedad utilizada en este experimento, cuando la planta esté próxima a una acumulación de grados días iguales o cercanos a 140 , se puede estimar que la planta a iniciado el macollamiento. No obstante, se debe de tomar en cuenta que esta variedad es de ciclo largo y existen variedades de ciclo más corto, por lo que los datos obtenidos no se deben aplicar para todas las variedades, se debe de calibrar para cada una de las variedades existentes principalmente las que son de mayor importancia para el productor. Conocer la relación que existe entre la planta y su entorno es fundamental para realizar un manejo técnico adecuado del cultivo. La información de sumatoria térmica permite planificar los controles fitosanitarios, la fecha de cosecha, o bien predecir el rendimiento esperado, además explica por qué fechas tan importantes como la floración no son las mismas entre diferentes ciclos de siembra de este cultivo.

\section{AGRADECIMIENTOS}

Los autores expresan su agradecimiento al Sr. Donald Stewart propietario de la Hacienda Mojica donde se realizó esta investigación. Al Ing. José María Agüero Gerente de la Compañía Agricultura Científica por la colaboración brindada durante el desarrollo de la investigación.

\section{LITERATURA CITADA}

ARNOLD C. 1960. Maximun-minimum temperatures as a basis for computing heat units. Journal of the American Society for Horticultural Sciences, Alexandria 76:682-692.

COUNCE P., KEISLING T., MITCHELL A. 2000. A Uniform, Objective and Adaptive System for Expressing Rice Development. Crop Sciencie 40:436-443.

DATTA S. 1986. Crecimiento y desarrollo de la planta de arroz. Producción de arroz. Fundamentos y Prácticas. Primera Edición. Editorial Limusa. 685 p.

HAUN J. 1973. Visual quantification of wheat development. Agron. J. 55:487-491.

IIRI. 1980. Standard evaluation system for rice. IRRI, Los Baños, Phillipines. 52 p.

LANCASHIRE P., BLEIHOLDER H., VAN DEN BOOM P., LANGELUDDEKE P., STAUSS E., WEBER E., WITZENBERGER. 1991. A uniform decimal code for growth stages of crops and weeds. Ann. Appl. Biol. 119:561-601.

LANGER R. 1972. How grasses grow. Edwuard Arnold, Ltda. London. 66 p.

MÉNDEZ R. 2002. Eventos fenológicos. Previsión de ocurrencia en el cultivo de arroz a través del modelo de suma térmica. Revista Arroz ACA 3(33):22-25.

MALDONADO I.B., CARAM G.A., ANGELONI P.N., FERNANDEZ C. 2006. Determinación del tiempo termal en la aparición de hojas de híbridos de girasol en Chaco. Cátedra de Agroclimatología, Facultad de Ciencias Agrarias, UNNE. Argentina. 5 p.

NISHIYAMA I. 1976. Effects of temperatura on the vegetative growth of rice plants. Climate and Rice, pp. 159-185. Proceedings of the symposium on climate and rice. International Rice Research Institute, Los Baños, Phillippines.

PASCALE A.J., DAMARIO E.A. 2004. Bioclimatología agrícola y Agroclimatología, pp. 184-210. In: Estimaciones Agroclimáticas de unidades calóricas Ed. Facultad de Agronomía. UBA.

SAUMELL H. 1980. Girasol: Técnicas actualizadas para su mejoramiento y cultivo, pp. 81-83. In: Editorial Hemisferio Sur S.A. Argentina.

STEINMETZ S. 2007. Impacto das mudancas climáticas globais sobre o arroz irrigado no Sul do Brasil, pp. 828-831. V Congresso Brasileiro de Arroz Irrigado. ANAIS XXVII Reuniao da Cultura do Arroz Irrigado. EMBRAPA Clima Temperado.

STEINMETZ S., FAGUNDEZ P., SCIVITTARO W., ULGUIM A., NOBRE F., PITANEL J., OLIVEIRA J., DA COSTA A. 2007. Estadios de desenvolvimiento de 12 cultivares de arroz irrigado expressos em graus-dia, pp. 368-369. V Congreso Brasileiro de arroz irrigado. Pelotas, Brasil. Anais, volumen I. 
STRECK N., WEISS A., XUE Q., BAENZIGER P., 2003. Improving predictions of developmental stages in winter wheat: A modified wang and Engel model. Agricultural and Forest Meteorology, Amsterdam 115(3-4):139-150.

VILLALOBOS R., RETANA J. 1997. Posibles efectos de un calentamiento global en el cultivo de arroz de secano en el pacífico norte de Costa Rica. Agronomía Costarricense 21(2):179-188.

YOSHIDA S. 1981. Fundaments of rice crop science. International Rice Research Institute, Los Baños, Phillipines p. 296.

ZADOKS J., CHANG T., KONZAK C. 1974. A decimal code for growth stages of cereals. Weed Res. 14:415-421.

Todos los derechos reservados. Universidad de Costa Rica. Este artículo se encuentra licenciado con Creative Commons Reconocimiento-NoComercial-SinObraDerivada 3.0 Costa Rica. Para mayor información escribir a rac.cia@ucr.ac.cr 
